\title{
Effective Spin Systems in Coupled Microcavities
}

\author{
Michael J. Hartmann, ${ }^{*}$ Fernando G. S. L. Brandão, and Martin B. Plenio \\ Institute for Mathematical Sciences, Imperial College London, SW7 2PG, United Kingdom \\ QOLS, The Blackett Laboratory, Imperial College London, Prince Consort Road, SW7 2BW, United Kingdom
}

(Received 14 May 2007; published 15 October 2007)

\begin{abstract}
We show that atoms trapped in microcavities that interact via the exchange of virtual photons can model an anisotropic Heisenberg spin-1/2 lattice in an external magnetic field. All parameters of the effective Hamiltonian can individually be tuned via external lasers. Since the occupations of excited atomic levels and photonic states are strongly suppressed, the effective model is robust against decoherence mechanisms, has a long lifetime, and its implementation is feasible with current experimental technology. The model provides a feasible way to create cluster states in these devices.
\end{abstract}

DOI: 10.1103/PhysRevLett.99.160501

PACS numbers: 03.67.Mn, 42.50.Vk, 75.10.Jm

Introduction. - Interacting spins or qubits are of central importance in quantum information processing (QIP) and condensed matter physics. Two or higher dimensional magnetic compounds are believed to host condensed matter phenomena, such as frustration and high $T_{c}$ superconductivity [1]. In quantum information, lattices of interacting spins can be employed to generate highly entangled states, such as cluster states which are the resource for one-way quantum computation [2].

While it is a prerequisite for QIP, single spin addressability can also be very helpful to obtain deeper and more detailed insight into condensed matter physics. In magnetic compounds where spin lattices appear naturally this is, however, extremely hard to achieve as the spatial separation between neighboring spins is very small and the time scales of interesting processes can be very short.

Here we show that effective spin lattices [3] can be generated with individual atoms in microcavities that are coupled to each other via the exchange of virtual photons. Because of the size and separation of the microcavities, individual lattice sites can be addressed with optical lasers, whereas the cavities can be arranged in arbitrary lattice geometries. The two spin polarizations $|\uparrow\rangle$ and $|\downarrow\rangle$ are represented by two long-lived atomic levels of a $\Lambda$ levelstructure (cf. Figs. 1 and 2). Together with external lasers, the cavity mode that couples to these atoms can induce Raman transitions between these two long-lived levels. With appropriately chosen detunings, both the excited atomic levels and photon states have vanishing occupation and can be eliminated from the description. As a result, the dynamics is confined to only two states per atom, the longlived levels, and can be described by a spin-1/2 Hamiltonian. Similar methods allow for the elimination of the atoms leading to quadrature squeezing of the field [4].

In our approach, the small occupation of photon states and excited atomic levels also strongly suppresses spontaneous emission and cavity decay. All these results are verified by detailed numerics. A realization of the scheme thus requires cavities that operate in a strong coupling regime with a high cooperativity factor and an atom photon coupling that exceeds cavity decay. Such regimes have now been achieved in several devices [5-7], making a realization of the presented scheme feasible with current technology. We begin by showing how to engineer effective $\sigma^{x} \sigma^{x}, \sigma^{y} \sigma^{y}$ and $\sigma^{z} \sigma^{z}$ interactions as well as the effective magnetic field $B \sigma_{z}$ and then explain how to generate the full anisotropic Heisenberg model. We verify the validity of all approximations by comparison with the exact dynamics of the full atom-cavity model and also apply the model to the generation of cluster states. Finally, we discuss the feasibility of our model for realistic experimental parameters.

$X X$ and $Y Y$ interactions. - We consider an array of cavities that are coupled via exchange of photons with one 3-level atom in each cavity (Fig. 1). Two long-lived levels, $|a\rangle$ and $|b\rangle$, represent the two spin states. The cavity mode couples to the transitions $|a\rangle \leftrightarrow|e\rangle$ and $|b\rangle \leftrightarrow|e\rangle$, where $|e\rangle$ is the excited state of the atom. Furthermore, two driving lasers couple to the transitions $|a\rangle \leftrightarrow|e\rangle$, respectively, $|b\rangle \leftrightarrow|e\rangle$. For the sake of simplicity we consider here a one-dimensional array. The generalization to higher dimensions is straightforward. The Hamiltonian of the atoms reads $H_{A}=\sum_{j=1}^{N} \omega_{e}\left|e_{j}\right\rangle\left\langle e_{j}\left|+\omega_{a b}\right| b_{j}\right\rangle\left\langle b_{j}\right|$, where

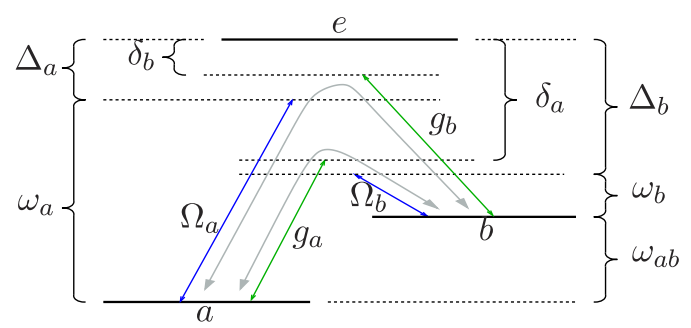

FIG. 1 (color online). Level structure, driving lasers, and relevant couplings to the cavity mode to generate effective $\sigma^{x} \sigma^{x}$ and $\sigma^{y} \sigma^{y}$-couplings for one atom. The cavity mode couples with strengths $g_{a}$ and $g_{b}$ to transitions $|a\rangle \leftrightarrow|e\rangle$ and $|b\rangle \leftrightarrow|e\rangle$, respectively. One laser with frequency $\omega_{a}$ couples to transition $|a\rangle \leftrightarrow|e\rangle$ with Rabi frequency $\Omega_{a}$ and another laser with frequency $\omega_{b}$ to $|b\rangle \leftrightarrow|e\rangle$ with $\Omega_{b}$. The dominant two-photon processes are indicated in faint gray arrows. 


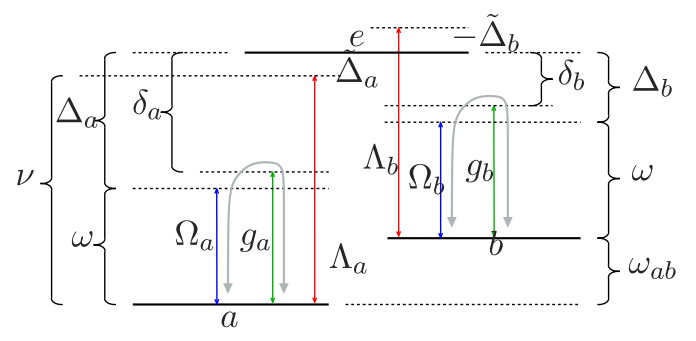

FIG. 2 (color online). Level structure, driving lasers, and relevant couplings to the cavity mode to generate effective $\sigma^{z} \sigma^{z}$-couplings for one atom. The cavity mode couples with strengths $g_{a}$ and $g_{b}$ to transitions $|a\rangle \leftrightarrow|e\rangle$ and $|b\rangle \leftrightarrow|e\rangle$, respectively. Two lasers with frequencies $\omega$ and $\nu$ couple with Rabi frequencies $\Omega_{a}$, respectively, $\Lambda_{a}$ to transition $|a\rangle \leftrightarrow|e\rangle$ and $\Omega_{b}$, respectively, $\Lambda_{b}$ to $|b\rangle \leftrightarrow|e\rangle$. The dominant two-photon processes are indicated in faint gray arrows.

the index $j$ counts the cavities, $\omega_{e}$ is the energy of the excited level and $\omega_{a b}$ the energy of level $|b\rangle$. The energy of level $|a\rangle$ is set to zero and we use $\hbar=1$. The Hamiltonian that describes the photons in the cavity modes is $H_{C}=$ $\omega_{C} \sum_{j=1}^{N} a_{j}^{\dagger} a_{j}+J_{C} \sum_{j=1}^{N}\left(a_{j}^{\dagger} a_{j+1}+a_{j} a_{j+1}^{\dagger}\right)$, where $a_{j}^{\dagger}$ creates a photon in cavity $j, \omega_{C}$ is the energy of the photons and $J_{C}$ the tunneling rate of photons between neighboring cavities [8]. For convenience we assume periodic boundary conditions, where $H_{C}$ can be diagonalized via a Fourier transform. Finally, the interaction between the atoms and the photons as well as the driving by the lasers are described by $H_{A C}=\sum_{j=1}^{N}\left[\left(\frac{1}{2} \Omega_{a} e^{-i \omega_{a} t}+g_{a} a_{j}\right)\left|e_{j}\right\rangle\left\langle a_{j}\right|+\right.$ H.c. $]+[a \leftrightarrow b]$. Here $g_{a}\left(g_{b}\right)$ are the couplings of the respective transitions to the cavity mode, $\Omega_{a}\left(\Omega_{b}\right)$ is the Rabi frequency of one laser with frequency $\omega_{a}\left(\omega_{b}\right)$ [9]. The complete Hamiltonian is then given by $H=H_{A}+$ $H_{C}+H_{A C}$.

We now switch to an interaction picture with respect to $H_{0}=H_{A}+H_{C}-\delta_{1} \sum_{j=1}^{N}\left|b_{j}\right\rangle\left\langle b_{j}\right|$, where $\delta_{1}=\omega_{a b}-$ $\left(\omega_{a}-\omega_{b}\right) / 2$, and adiabatically eliminate the excited atom levels $\left|e_{j}\right\rangle$ and the photons [10]. We consider terms up to 2nd order in the effective Hamiltonian and drop fast oscillating terms. For this approach the detunings $\Delta_{a} \equiv$ $\omega_{e}-\omega_{a}, \Delta_{b} \equiv \omega_{e}-\omega_{b}-\left(\omega_{a b}-\delta_{1}\right), \quad \delta_{a}^{k} \equiv \omega_{e}-\omega_{k}$ and $\delta_{b}^{k} \equiv \omega_{e}-\omega_{k}-\left(\omega_{a b}-\delta_{1}\right)$ have to satisfy $\left|\Delta_{a}\right|$, $\left|\Delta_{b}\right|,\left|\delta_{a}^{k}\right|,\left|\delta_{b}^{k}\right| \gg\left|\Omega_{a}\right|,\left|\Omega_{b}\right|,\left|g_{a}\right|,\left|g_{b}\right|$ (for all $k$ ). Furthermore, the parameters must be such that the dominant Raman transitions between levels $a$ and $b$ are those that involve one laser photon and one cavity photon each (cf. Fig. 1). To avoid excitations of real photons via these transitions, we furthermore require $\left|\Delta_{a}-\delta_{b}^{k}\right|, \mid \Delta_{b}-$ $\delta_{a}^{k}|\gg| \frac{\Omega_{a} g_{b}}{2 \Delta_{a}}|,| \frac{\Omega_{b} g_{a}}{2 \Delta_{b}} \mid$ (for all $k$ ).

Hence, whenever the atom emits or absorbs a virtual photon into or from the cavity mode, it does a transition from level $|a\rangle$ to $|b\rangle$ or vice versa. If an atom emits a virtual photon that is absorbed by a neighboring atom, which then also does a transition between $|a\rangle$ to $|b\rangle$, an effective spin- spin interaction arises. Dropping irrelevant constants, the resulting effective Hamiltonian reads $H_{x y}=\sum_{j=1}^{N} B \sigma_{j}^{z}+$ $\left(J_{1} \sigma_{j}^{+} \sigma_{j+1}^{-}+J_{2} \sigma_{j}^{-} \sigma_{j+1}^{-}+\right.$H.c. $)$, where $\sigma_{j}^{z}=\left|b_{j}\right\rangle \times$ $\left\langle b_{j}|-| a_{j}\right\rangle\left\langle a_{j}\right|$ and $\sigma_{j}^{+}=\left|b_{j}\right\rangle\left\langle a_{j}\right| . B, J_{1}$, and $J_{2}$ are given to second order by [11]. If $J_{2}^{\star}=J_{2}$, this Hamiltonian reduces to the $X Y$ model,

$$
H_{x y}=\sum_{j=1}^{N} B \sigma_{j}^{z}+J_{x} \sigma_{j}^{x} \sigma_{j+1}^{x}+J_{y} \sigma_{j}^{y} \sigma_{j+1}^{y},
$$

with $J_{x}=\left(J_{1}+J_{2}\right) / 2$ and $J_{y}=\left(J_{1}-J_{2}\right) / 2$. For $\Omega_{a}=$ $\pm\left(\Delta_{a} g_{a} / \Delta_{b} g_{b}\right) \Omega_{b}$ with $\Omega_{a}$ and $\Omega_{b}$ real, the interaction is either purely $\sigma^{x} \sigma^{x}(+)$ with $J_{y}=0$ or purely $\sigma^{y} \sigma^{y}(-)$ with $J_{x}=0$ and the Hamiltonian (1) becomes the Ising model in a transverse field, whereas the isotropic $X Y$ model $\left(J_{x}=J_{y}\right.$, i.e., $\left.J_{2}=0\right)$ [12] is obtained for either $\Omega_{a} \rightarrow 0$ or $\Omega_{b} \rightarrow 0$. The effective magnetic field $B$ in turn can, independently of $J_{x}$ and $J_{y}$, be tuned to assume any value between $|B| \gg\left|J_{x}\right|,\left|J_{y}\right|$ and $|B| \ll\left|J_{x}\right|,\left|J_{y}\right|$ by varying $\delta_{1}$. Thus we will be able to drive the system through a quantum phase transition. Now we proceed to effective $Z Z$ interactions.

ZZ interactions. - To obtain an effective $\sigma^{z} \sigma^{z}$ interaction, we again use the same atomic level configuration but now only one laser with frequency $\omega$ mediates atomatom coupling via virtual photons. A second laser with frequency $\nu$ is used to tune the effective magnetic field via a Stark shift. The atoms together with their couplings to cavity mode and lasers are shown in Fig. 2. Again, we consider the one-dimensional case as an example. The generalization to higher dimensions is straight forward. The Hamiltonians $H_{A}$ of the atoms and $H_{C}$ of the cavity modes thus have the same form as above, whereas $H_{A C}$ now reads $H_{A C}=\sum_{j=1}^{N}\left[\left(\frac{\Omega_{a}}{2} e^{-i \omega t}+\frac{\Lambda_{a}}{2} e^{-i \nu t}+\right.\right.$ $\left.g_{a} a_{j}\right)\left|e_{j}\right\rangle\left\langle a_{j}\right|+$ H.c. $]+[a \leftrightarrow b]$. Here, $\Omega_{a}$ and $\Omega_{b}$ are the Rabi frequencies of the driving laser with frequency $\omega$ on transitions $|a\rangle \rightarrow|e\rangle$ and $|b\rangle \rightarrow|e\rangle$, whereas $\Lambda_{a}$ and $\Lambda_{b}$ are the Rabi frequencies of the driving laser with frequency $\nu$ on transitions $|a\rangle \rightarrow|e\rangle$ and $|b\rangle \rightarrow|e\rangle$.

We switch to an interaction picture with respect to $H_{0}=$ $H_{A}+H_{C}$ and adiabatically eliminate the excited atom levels $\left|e_{j}\right\rangle$ and the photons [10]. Again, the detunings $\Delta_{a} \equiv$ $\omega_{e}-\omega, \quad \Delta_{b} \equiv \omega_{e}-\omega-\omega_{a b}, \quad \tilde{\Delta}_{a} \equiv \omega_{e}-\nu, \quad \tilde{\Delta}_{b} \equiv$ $\omega_{e}-\nu-\omega_{a b}, \delta_{a}^{k} \equiv \omega_{e}-\omega_{k}$ and $\delta_{b}^{k} \equiv \omega_{e}-\omega_{k}-\omega_{a b}$ have to be large compared to the couplings $\Omega_{a}, \Omega_{b}, \Lambda_{a}$, $\Lambda_{b}, g_{a}$ and $g_{b}$, i.e. $\left|\Delta_{a}\right|,\left|\Delta_{b}\right|,\left|\delta_{a}^{k}\right|,\left|\delta_{b}^{k}\right| \gg\left|\Omega_{a}\right|,\left|\Omega_{b}\right|$, $\left|g_{a}\right|,\left|g_{b}\right|$ and $\left|\tilde{\Delta}_{a}\right|,\left|\tilde{\Delta}_{b}\right| \gg\left|\Lambda_{a}\right|,\left|\Lambda_{b}\right|$ (for all $k$ ), whereas now Raman transitions between levels $a$ and $b$ should be suppressed. Hence, parameters must be such that the dominant two-photon processes are those that involve one laser photon and one cavity photon each but where the atom does no transition between levels $a$ and $b$ (cf. Fig. 2). To avoid excitations of real photons in these processes, we 
thus require $\left|\Delta_{a}-\delta_{a}^{k}\right|,\left|\Delta_{b}-\delta_{b}^{k}\right| \gg\left|\frac{\Omega_{a} g_{a}}{2 \Delta_{a}}\right|,\left|\frac{\Omega_{b} g_{b}}{2 \Delta_{b}}\right|$ (for all $k$ ).

Whenever two atoms exchange a virtual photon in this scheme, both experience a Stark shift that depends on the state of the partner atom. This conditional Stark shift plays the role of an effective $\sigma^{z} \sigma^{z}$-interaction. Dropping irrelevant constants, the effective Hamiltonian reads

$$
H_{z z}=\sum_{j=1}^{N}\left(\tilde{B} \sigma_{j}^{z}+J_{z} \sigma_{j}^{z} \sigma_{j+1}^{z}\right) .
$$

$\tilde{B}$ and $J_{z}$ are given to second order by [13] and can be tuned independently, either by varying $\Omega_{a}$ and $\Omega_{b}$ for $J_{z}$ or by varying $\Lambda_{a}$ and $\Lambda_{b}$ for $\tilde{B}$. In particular, $\left|\Lambda_{a}\right|^{2}$ and $\left|\Lambda_{b}\right|^{2}$ can for all values of $\Omega_{a}$ and $\Omega_{b}$ be chosen such that either $J_{z} \ll \tilde{B}$ or $J_{z} \gg \tilde{B}$.

The complete effective model.-Making use of the Suzuki-Trotter formula, the two Hamiltonians (1) and (2) can now be combined to one effective Hamiltonian. To this end, the lasers that generate the Hamiltonian (1) are turned on for a short time interval $d t\left(\left\|H_{x y}\right\| \cdot d t \ll 1\right)$ followed by another time interval $d t\left(\left\|H_{z z}\right\| \cdot d t \ll 1\right)$ with the lasers that generate the Hamiltonian (2) turned on. This sequence is repeated until the total time range to be simulated is covered. The effective Hamiltonian simulated by this procedure is $H_{\text {spin }}=H_{x y}+H_{z z}$ or

$$
H_{\mathrm{spin}}=\sum_{j=1}^{N}\left(B_{\mathrm{tot}} \sigma_{j}^{z}+\sum_{\alpha=x, y, z} J_{\alpha} \sigma_{j}^{\alpha} \sigma_{j+1}^{\alpha}\right),
$$

where $B_{\text {tot }}=B+\tilde{B}$. The time interval $d t$ should thereby be chosen such that $\Omega^{-1}, g^{-1} \ll d t_{1}, d t_{2} \ll J_{x}^{-1}, J_{y}^{-1}$, $J_{z}^{-1}, B^{-1}$ and $\tilde{B}^{-1}$, so that the Trotter sequence concatenates the effective Hamiltonians $H_{X Y}$ and $H_{Z Z}$. The procedure can be generalized to higher order Trotter formulas or by turning on the sets of lasers for time intervals of different length.

Numerical tests. - To confirm the validity of our approximations, we numerically simulate the dynamics generated


FIG. 3. The occupation probability $p\left(a_{1}\right)$ of state $\left|a_{1}\right\rangle$ (solid line) and the probability $p\left(\downarrow_{1}\right)$ of spin 1 to point down (dashed line) for the parameters $\omega_{e}=10^{6} \mathrm{GHz}, \omega_{a b}=30 \mathrm{GHz}, \Delta_{a}=$ $30 \mathrm{GHz}, \quad \Delta_{b}=60 \mathrm{GHz}, \quad \omega_{C}=\omega_{e}-\Delta_{b}+2 \mathrm{GHz}, \quad \tilde{\Delta}_{a}=$ $15 \mathrm{GHz}, \quad \Omega_{a}=\Omega_{b}=2 \mathrm{GHz}, \quad \Lambda_{a}=\Lambda_{b}=0.71 \mathrm{GHz}, \quad g_{a}=$ $g_{b}=1 \mathrm{GHz}, J_{C}=0.2 \mathrm{GHz}$, and $\delta_{1}=-0.0165 \mathrm{GHz}$ (a) respectively $\delta_{1}=-0.0168 \mathrm{GHz}(\mathrm{b})$. Both, the occupation of the excited atomic states $\left\langle\mid e_{j}\right\rangle\left\langle e_{j} \mid\right\rangle$ and the photon number $\left\langle a^{\dagger} a\right\rangle$ are always smaller than 0.03 . by the full Hamiltonian $H$ and compare it to the dynamics generated by the effective model (3).

As an example we consider two atoms in two cavities, initially in the state $\frac{1}{\sqrt{2}}\left(\left|a_{1}\right\rangle+\left|b_{1}\right\rangle\right) \otimes\left|a_{2}\right\rangle$, and calculate the occupation probability $p\left(a_{1}\right)$ of the state $\left|a_{1}\right\rangle$ which corresponds to the probability of spin 1 to point down, $p\left(\downarrow_{1}\right)$. Figure 3(a) shows $p\left(a_{1}\right)$ and $p\left(\downarrow_{1}\right)$ for an effective Hamiltonian (3) with $B_{\text {tot }}=0.135 \mathrm{MHz}, J_{x}=$ $0.065 \mathrm{MHz}, J_{y}=0.007 \mathrm{MHz}$ and $J_{z}=0.004 \mathrm{MHz}$ and hence $\left|B_{\text {tot }}\right|>\left|J_{x}\right|$, whereas Fig. 3(b) shows $p\left(a_{1}\right)$ and $p\left(\downarrow_{1}\right)$ for an effective Hamiltonian (3) with the same $J_{x}$, $J_{y}$ and $J_{z}$ but $B_{\text {tot }}=-0.025 \mathrm{MHz}$ and hence $\left|B_{\text {tot }}\right|<\left|J_{x}\right|$ [14]. Discrepancies between numerical results for the full and the effective model are due to higher order terms for the parameters $B, \tilde{B}, J_{x}, J_{y}$, and $J_{z}$, which lead to relative corrections of up to $10 \%$ in the considered cases. Let us stress here that despite this lack of accuracy of the second order approximations, the effective model is indeed a spin-1/2 Hamiltonian as occupations of excited atomic and photon states are negligible.

Cluster state generation. - The Hamiltonian (2) can be used to generate cluster states $[2,15]$. To this end, all atoms are initialized in the states $\left(\left|a_{j}\right\rangle+\left|b_{j}\right\rangle\right) / \sqrt{2}$, which can be done via a STIRAP process [16], and then evolved under the Hamiltonian (2) for $t=\pi / 4 J_{z}$. Figure 4 shows the von Neumann entropy of the reduced density matrix of one effective spin $E_{\mathrm{vN}}$ and the purity of the reduced density matrix of the effective spin chain $P_{s}$ for a full three cavity model. Since $E_{\mathrm{vN}} \approx \log _{2} 2$ for $t \approx 50 \mu$ s while the state of the effective spin model remains highly pure $\left(P_{s}=\right.$ $\left.\operatorname{tr}\left[\rho^{2}\right]>0.995\right)$ the degree of entanglement will be very close to maximal, see e.g. [17]. Thus the levels $\left|a_{j}\right\rangle$ and $\left|b_{j}\right\rangle$ have indeed been driven into a state which is, up to local unitary rotations, very close to a three-qubit cluster state.

Experimental implementation.-For an experimental implementation, the parameters of the effective Hamiltonian, $J_{x}, J_{y}, J_{z}, B$, and $\tilde{B}$ have to be much larger than rates for decay mechanisms via the photons or the
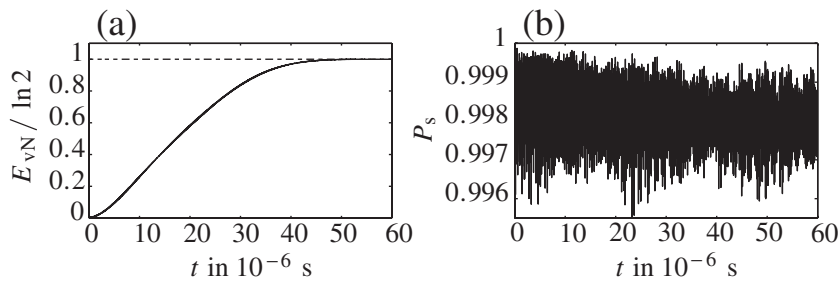

FIG. 4. (a) The von Neumann entropy $E_{\mathrm{vN}}$ of the reduced density matrix of 1 effective spin in multiples of $\ln 2$ and (b) the purity of the reduced state of the effective spin model for 3 cavities where $J_{z}=0.021 \mathrm{MHz}$. The plots assume that no spontaneous emission took place. For a spontaneous emission rate of $0.1 \mathrm{MHz}(g=1 \mathrm{GHz})$, the probability for a decay event in the total time range is $1.5 \%$. Hence, cluster state generation fails with probability $0.005 n$ for $n \ll 1 / 0.005=200$ cavities, irrespective of the lattice dimension. 
excited states $\left|e_{j}\right\rangle$. With the definitions $\Omega=\max \left(\Omega_{a}, \Omega_{b}\right)$, $g=\max \left(g_{a}, g_{b}\right), \Delta=\min \left(\Delta_{a}, \Delta_{b}\right)$, the occupation of the excited levels $\left|e_{j}\right\rangle$ and the photon number $n_{p}$ can be estimated to be $\left\langle\mid e_{j}\right\rangle\left\langle e_{j} \mid\right\rangle \approx|\Omega / 2 \Delta|^{2}$ and $n_{p} \approx$ $\left|(\Omega g / 2 \Delta) \gamma_{1}\right|^{2}$, whereas the couplings $J_{x}, J_{y}$ and $J_{z}$ are approximately $|(\Omega g / 2 \Delta)|^{2} \gamma_{2}$. Spontaneous emission from the levels $\left|e_{j}\right\rangle$ at a rate $\Gamma_{E}$ and cavity decay of photons at a rate $\Gamma_{C}$ thus lead to effective decay rates $\Gamma_{1}=|\Omega / 2 \Delta|^{2} \Gamma_{E}$ and $\Gamma_{2}=\left|(\Omega g / 2 \Delta) \gamma_{1}\right|^{2} \Gamma_{C}$. Hence, we require $\Gamma_{1} \ll$ $|(\Omega g / 2 \Delta)|^{2} \gamma_{2}$ and $\Gamma_{2} \ll|(\Omega g / 2 \Delta)|^{2} \gamma_{2}$ which implies $\Gamma_{E} \ll J_{C} g^{2} / \delta^{2}$ and $\Gamma_{C} \ll J_{C}\left(J_{C}<\delta / 2\right)$, where, $\delta=$ $\left|\left(\omega_{a}+\omega_{b}\right) / 2-\omega_{C}\right|$ for the $X X$ and $Y Y$ interactions and $\delta=\left|\omega-\omega_{C}\right|$ for the $Z Z$ interactions and we have approximated $\left|\gamma_{1}\right| \approx \delta^{-1}$ and $\left|\gamma_{2}\right| \approx J_{C} \delta^{-2}$. Since photons should be more likely to tunnel to the next cavity than decay into free space, $\Gamma_{C} \ll J_{C}$ should hold in most cases. For $\Gamma_{E} \ll J_{C} g^{2} / \delta^{2}$, to hold, cavities with a high ratio $g / \Gamma_{E}$ are favorable. Since $\delta>2 J_{C}$, the two requirements together imply that the cavities should have a high cooperativity factor.

This regime can be achieved in microcavities, which have a small volume and thus a high $g$. Suitable candidates for the present proposal are, for example, photonic band gap cavities which can either couple to atoms or quantum dots. Here, cooperativity factors of $g^{2} / 2 \Gamma_{C} \Gamma_{E} \sim 100$ and values of $g / \Gamma_{E} \sim 300$ (off resonance) have been realized [6] and $g^{2} / 2 \Gamma_{C} \Gamma_{E} \sim 10^{5}$, respectively, $g / \Gamma_{E} \sim 10^{5}$ are predicted to be achievable [18]. Further promising devices are microcavities on a gold coated silicon chip that couple to single trapped atoms, where $g^{2} / 2 \Gamma_{C} \Gamma_{E} \sim 40$ and $g / \Gamma_{E} \sim 50$ have been achieved [7]. Both are fabricated in large arrays and couple via the overlap of their evanescent fields or optical fibers that transfer photons from one cavity to another. Depending on the technical realization of the photon tunneling, one-, two- or three-dimensional spin lattices can be created.

Summary. - We have shown that single atoms in interacting cavities that are operated in a strong coupling regime can form a Heisenberg spin-1/2 Hamiltonian. All parameters of the effective Hamiltonian can be tuned individually, making the device a universal simulator for this model. When operated in a two-dimensional array of cavities the device is thus able to simulate spin lattices which are not trackable with numerics on classical computers. Furthermore, this system can be used to generate cluster states on such lattices. Together with the possibility to measure individual lattice sites it thus provides the two key requirements for one-way quantum computation. This demonstrates the versatility of the present setup for the control and manipulation of quantum systems in parameter ranges that are experimentally accessible.

This work is part of the QIP-IRC supported by EPSRC (No. GR/S82176/0), the Integrated Project Qubit Appli- cations (QAP) supported by the IST directorate as Contract Number No. 015848 and was supported by the EPSRC Grant No. EP/E058256, the Alexander von Humboldt Foundation, the Conselho Nacional de Desenvolvimento Científico e Tecnológico (CNPq) and the Royal Society.

*m.hartmann@imperial.ac.uk

[1] T. Moriya and K. Ueda, Rep. Prog. Phys. 66, 1299 (2003).

[2] H. J. Briegel and R. Raussendorf, Phys. Rev. Lett. 86, 910 (2001); R. Raussendorf and H. J. Briegel, Phys. Rev. Lett. 86, 5188 (2001); D. Gross and J. Eisert, Phys. Rev. Lett. 98, 220503 (2007).

[3] F. Mintert and C. Wunderlich, Phys. Rev. Lett. 87, 257904 (2001); D. Porras and J. I. Cirac, Phys. Rev. Lett. 92, 207901 (2004).

[4] R. Guzmán et al., Phys. Rev. Lett. 96, 010502 (2006).

[5] T. Aoki et al., Nature (London) 443, 671 (2006).

[6] A. Badolato et al., Science 308, 1158 (2005); K. Hennessy et al., Nature (London) 445, 896 (2007); B.-S. Song et al., Nat. Mater. 4, 207 (2005).

[7] M. Trupke et al., Appl. Phys. Lett. 87, 211106 (2005).

[8] M. J. Hartmann, F. G. S. L. Brandão, and M. B. Plenio, Nature Phys. 2, 849 (2006); M. J. Hartmann and M. B. Plenio, Phys. Rev. Lett. 99, 103601 (2007).

[9] A. Søndberg Sørensen and K. Mølmer, Phys. Rev. A 66, 022314 (2002); F. Dimer et al., Phys. Rev. A 75, 013804 (2007).

[10] D. F. V. James, Fortschr. Phys. 48, 823 (2000).

[11] $B=\frac{\delta_{1}}{2}-\frac{1}{2}\left[\frac{\left|\Omega_{b}\right|^{2}}{4 \Delta_{b}^{2}}\left(\Delta_{b}-\frac{\left|\Omega_{b}\right|^{2}}{4 \Delta_{b}}-\frac{\left|\Omega_{a}\right|^{2}}{4\left(\Delta_{a}-\Delta_{b}\right)}-\gamma_{b} g_{b}^{2}-\gamma_{1} g_{a}^{2}+\right.\right.$ $\left.\left.\gamma_{1}^{2} \frac{g_{a}^{4}}{\Delta_{b}}\right)-(a \leftrightarrow b)\right], \quad J_{1}=\frac{\gamma_{2}}{4}\left(\frac{\left|\Omega_{a}\right|^{2} g_{b}^{2}}{\Delta_{a}^{2}}+\frac{\left|\Omega_{b}\right|^{2} g_{a}^{2}}{\Delta_{b}^{2}}\right)$ and $J_{2}=$ $\frac{\gamma_{2}}{2} \frac{\Omega_{a}^{\star} \Omega_{b} g_{a} g_{b}}{\Delta_{a} \Delta_{b}}, \quad$ where $\quad \gamma_{a, b}=\frac{1}{N} \sum_{k} \frac{1}{\omega_{a, b}-\omega_{k}}, \quad \gamma_{1}=$ $\frac{1}{N} \sum_{k} \frac{1}{\left(\omega_{a}+\omega_{b}\right) / 2-\omega_{k}}$ and $\gamma_{2}=\frac{1}{N} \sum_{k} \frac{\exp (i k)}{\left(\omega_{a}+\omega_{b}\right) / 2-\omega_{k}}$.

[12] L.-M. Duan, E. Demler, and M. D. Lukin, Phys. Rev. Lett. 91, 090402 (2003); D. G. Angelakis, M. F. Santos, and S. Bose, arXiv:quant-ph/0606159 [Phys. Rev. A (to be published)].

[13] $J_{z}=\gamma_{2}\left|\frac{\Omega_{b}^{\star} g_{b}}{4 \Delta_{b}}-\frac{\Omega_{a}^{\star} g_{a}}{4 \Delta_{a}}\right|^{2}$ and $\tilde{B}=-\frac{1}{2}\left[\frac{\left|\Lambda_{b}\right|^{2}}{16 \tilde{\Delta}_{b}^{2}}\left(4 \tilde{\Delta}_{b}-\frac{\left|\Lambda_{a}\right|^{2}}{\tilde{\Delta}_{a}-\tilde{\Delta}_{b}}-\right.\right.$ $\left.\frac{\left|\Lambda_{b}\right|^{2}}{\tilde{\Delta}_{b}}-\sum_{j=a, b}\left(\frac{\left|\Omega_{j}\right|^{2}}{\Delta_{j}-\tilde{\Delta}_{b}}+4 \tilde{\gamma}_{j b} g_{j}^{2}\right)\right)+\frac{\left|\Omega_{b}\right|^{2}}{16 \Delta_{b}^{2}}\left(4 \Delta_{b}-\frac{\left|\Omega_{a}\right|^{2}}{\Delta_{a}-\Delta_{b}}-\right.$ $\left.\left.\frac{\left|\Omega_{b}\right|^{2}}{\Delta_{b}}-\sum_{j=a, b}\left(\frac{\left|\Lambda_{j}\right|^{2}}{\tilde{\Delta}_{j}-\Delta_{b}}+4 \gamma_{j b} g_{j}^{2}\right)+4 \gamma_{b b}^{2} \frac{g_{b}^{4}}{\Delta_{b}}\right)-(a \leftrightarrow b)\right]$ and with $\quad \gamma_{1}=\frac{1}{N} \sum_{k} \frac{1}{\omega-\omega_{k}}, \quad \gamma_{2}=\frac{1}{N} \sum_{k} \frac{\exp (i k)}{\omega-\omega_{k}}, \quad \gamma_{a a}=$ $\left.\left.\gamma_{b b}=\frac{1}{N} \sum_{k} \frac{1}{\omega-\omega_{k}}, \quad \gamma_{b a}\right\}=\frac{1}{N} \sum_{k} \frac{1}{\omega \pm \omega_{a b}-\omega_{k}}, \quad \tilde{\gamma}_{b a}\right\}=$ $\frac{1}{N} \sum_{k} \frac{1}{\nu \pm \omega_{a b}-\omega_{k}}, \tilde{\gamma}_{a a}=\tilde{\gamma}_{b b}=\frac{1}{N} \sum_{k} \frac{1}{\nu-\omega_{k}}$.

[14] M. J. Hartmann, M.E. Reuter, and M.B. Plenio, New J. Phys. 8, 94 (2006).

[15] D. G. Angelakis and A. Kay, arXiv:quant-ph/0702133v1.

[16] M. Fleischhauer, A. Imamoğlu, and J. P. Marangos, Rev. Mod. Phys. 77, 633 (2005).

[17] K. M. R. Audenaert and M. B. Plenio, New J. Phys. 8, 266 (2006).

[18] S. M. Spillane et al., Phys. Rev. A 71, 013817 (2005). 